\title{
Topoisomerase I and II $\alpha$ protein expression in primary colorectal cancer and recurrences following 5-fluorouracil-based adjuvant chemotherapy
}

\author{
Nicolas Tsavaris • Andreas Lazaris • Christos Kosmas • Panagiotis Gouveris • \\ Nikolaos Kavantzas • Petros Kopterides - Thomas Papathomas • George Agrogiannis • \\ Haralambos Zorzos $\cdot$ Vassiliki Kyriakou $\cdot$ Efstratios Patsouris
}

Published online: 27 March 2009

(C) Springer-Verlag 2009

Erratum to: Cancer Chemother Pharmacol

DOI 10.1007/s00280-008-0886-4

Unfortunately, two author names were published incorrect in the online version of the article. Correct names are given in the bold version. Instead of Nicolas Tsavaris, Andreas Lazaris, Christos Kosmas, Panagiotis Gouveris, Nikolaos Kavantzas, Petros Kopterides, Thomas Papathomas, George Arapogiannis, Haralambos Zorzos, Vassiliki Kyriakou, Efstathios Patsouris it should read as Nicolas Tsavaris, Andreas Lazaris, Christos Kosmas, Panagiotis Gouveris, Nikolaos Kavantzas, Petros Kopterides, Thomas Papathomas, George Agrogiannis, Haralambos Zorzos, Vassiliki Kyriakou, Efstratios Patsouris.

The online version of the original article can be found under doi:10.1007/s00280-008-0886-4.

N. Tsavaris $(\varangle) \cdot$ P. Gouveris $\cdot$ P. Kopterides

Medical Oncology Unit, Department of Pathophysiology,

Medical School, "Laikon” University General Hospital,

National and Kapodistrian University of Athens,

Athens University School of Medicine, 11527 Athens, Greece

e-mail: tsavari1@otenet.gr

A. Lazaris · N. Kavantzas · T. Papathomas · G. Agrogiannis ·

H. Zorzos $\cdot$ V. Kyriakou $\cdot$ E. Patsouris

First Department of Pathology, Medical School,

"Laikon" University General Hospital, National and Kapodistrian,

University of Athens, Athens, Greece

C. Kosmas

Second Department of Medical Oncology,

"Metaxa" Cancer Hospital, Piraeus, Greece

e-mail: ckosm1@ath.forthnet.gr 